\title{
ON THE COUNTING FUNCTION FOR THE $a$-VALUES OF A MEROMORPHIC FUNCTION
}

\author{
SAKARI TOPPILA
}

\section{Introduction and results}

1. We use the usual notation of the Nevanlinna theory. We let $\Sigma$ denote the Riemann sphere. For any function $f$ in the plane let $n(r)=$ $\sup _{a \in \Sigma} n(r, a)$ be the maximum number of roots of the equation $f(z)=a$ in $|z| \leq r$, and let $A(r)$ be the average value of $n(r, a)$ as $a$ moves over the Riemann sphere. Hayman [2] has proved that

$$
1 \leq \liminf _{r \rightarrow \infty} n(r) / A(r) \leq e .
$$

We shall consider the following problem of Hayman [3, Problem 1.16]: Can $e$ in (1) be replaced by any smaller quantity and in particular by 1 ? We shall show by an example that $e$ here cannot be replaced by 1 . and

Theorem 1. Let $h(z)=4(z-1) /(3 z), g_{n}(w)=1-(1 / w)^{2^{n}}$,

$$
f(z)=\prod_{p=1}^{\infty} \frac{g_{2 p-1}\left(h\left(-z / 3^{2 p-1}\right)\right)}{g_{2 p}\left(h\left(z / 3^{2 p}\right)\right)} .
$$

Then $f$ satisfies the condition $\lim \inf _{r \rightarrow \infty} n(r) / A(r) \geq 80 / 79$.

2. Next we shall consider the following problem of Erdös (Hayman [1, Problem 1.25]): Does there exist a meromorphic function such that for every pair of distinct values $a, b$ we have $\lim \sup _{r \rightarrow \infty} n(r, a) / n(r, b)=\infty$ ? We prove the following theorems.

Th e o r e m 2. There exists a meromorphic function $f$ such that for every pair $a, b, a \neq b$, we have $\lim \sup _{r \rightarrow \infty} n(r, a) / n(r, b)=\infty$.

$\mathrm{Th}$ e o r e m 3. There exists an entire function $f$ such that for every pair of distinct finite values $a$ and $b, \lim \sup _{r \rightarrow \infty} n(r, a) / n(r, b)=\infty$. 
3. If $a \in \Sigma$ and $b \in \Sigma$, the distance between $a$ and $b$ is defined to be the length of the shorter great circle arc on $\Sigma$ joining $a$ and $b$. This distance is denoted by $\delta(a, b)$. If $E \subset[1, \infty)$, we denote the logarithmic measure of $E$ by $m_{1}(E)=\int_{E} d t / t$. If $E_{r}=E \cap[1, r]$, by the lower logarithmic density of $E$ we mean $\lim \inf _{r \rightarrow \infty} m_{1}\left(E_{r}\right) / \log r$. Hayman and Stewart [4] have proved the following

$\mathrm{Th}$ e o r e m A. If $f$ is meromorphic in $|z|<\infty$ and $\varepsilon>0$, there exists a set $E$ of $r$-values having positive lower logarithmic density on which $n(r)<(1+\varepsilon) e A(r)$.

Miles [5] has proved the following

Th e o r e m B. There exist absolute constants $K<\infty$ and $C \in(0,1)$ such that if $f$ is any nonconstant meromorphic function in $|z|<\infty$, there exists $E \subset[1, \infty)$ having lower logarithmic density at least $C$ with the property that, if $\varepsilon>0$, there exists $r_{0}=r_{0}(\varepsilon)$ such that if $a_{1}, \ldots, a_{q}$ are elements of $\Sigma$ with $\delta\left(a_{i}, a_{j}\right) \geq \varepsilon$ for $i \neq j$, then

$$
\sum_{j=1}^{q}\left|n\left(r, a_{j}\right)\right| A(r)-1 \mid<K
$$

for all $r \in E, \quad r>r_{0}(\varepsilon)$.

We shall show by an example that the characterization of the set $E$ in Theorem A and in Theorem B is the best possible in the sense that the exceptional set of $r$-values may have positive lower logarithmic density.

$\mathrm{Th}$ e or e m 4. Let $s>10$ be an integer. The function

$$
f(z)=\prod_{n=1}^{\infty}\left(1-z \exp \left\{-(2 s)^{n}\right\}\right)^{(-s)^{n}}
$$

satisfies the condition $n(r, 0) / A\left(r^{1+1 /(5 s)}\right)>s / 2$ in a set $B$ having lower logarithmic density at least $(2 s)^{-4}$.

4. For $B \subset \Sigma$, we denote $n(r, B)=\sup _{w \in B} n(r, w)$. Rickman [6] has proved the following result.

The or e $\mathrm{m}$ C. Given $M>1$, there exists $K>1$ such that if $f$ is meromorphic in the plane with at least one asymptotic value $a$ and $B$ is a compact subset of $\Sigma$ not containing the point $a$, then

$$
\lim \sup n(r, B) / A(K r) \leq M \text {. }
$$

We shall show by an example that if $M<9 / 5$, the constant $K$ in (2) cannot be replaced by 1 .

Theorem 5. Let $t_{0}=100$ and for $n \geq 1$ let $t_{n}=4^{t_{n-1}}$ and $\log r_{n}=\left(t_{n} / t_{n-1}\right) \log (6 / 5)$. The entire function

$$
f(z)=\prod_{n=1}^{\infty}\left(1-z / r_{n}\right)^{t_{n}}
$$


satisfies the condition $\limsup _{r \rightarrow \infty} n(r, 0) / A(7 r / 6) \geq 9 / 5$.

Furthermore, we shall show that the set $B$ in (2) cannot be replaced by $\Sigma$.

Theorem 6. Let $t_{1}=100$ and $t_{n}=2^{t_{n-1}}$ for $n \geq 2$, and let $\log r_{n}=t_{n}$. The entire function

$$
f(z)=\prod_{n=1}^{\infty}\left(1+z / r_{n}\right)^{t_{n}}
$$

satisfies the condition $\lim \sup _{r \rightarrow \infty} n(r) / A(K r)=\infty$ for every constant $K \geq 1$.

\section{Proofs}

5. Proof of Theorem 1. We denote $r_{n}=3^{n}$. The function $h$ maps the circle $|z-16 / 7|=12 / 7$ onto the circle $|w|=1$. Therefore we see easily that

$$
f(z) \rightarrow A=\prod_{p=1}^{\infty} \frac{1-(3 / 4)^{2^{2 p-1}}}{1-(3 / 4)^{2^{2 p}}}
$$

as $z \rightarrow \infty$ outside the union of the discs

$$
C_{n}=\left\{z:\left|z-16(-1)^{n} r_{n}\right| 7 \mid<25 r_{n} / 14\right\} \text {. }
$$

Let $n_{p}(r, a)$ be the number of roots of the equation $f(z)=a$ in $C_{p} \cap$ $\{z:|z| \leq r\}$. We denote by $D_{\varepsilon}$ the union of the discs $|w|<\varepsilon$, $|w-A|<\varepsilon$ and $|w|>1 / \varepsilon$. Let $\varepsilon>0$. It follows from Rouché's theorem that $n_{p}\left(5 r_{p}, a\right)=2^{p}$ for all large values of $p$ and all $a \notin D_{\varepsilon}$.

From the properties of the function $g_{n}$ we see that if $p$ is sufficiently large, say $p \geq p_{\varepsilon}$, then

$$
n_{2 p}\left(3 r_{2 p}, \infty\right)<(19 / 20) 2^{2 p}
$$

and if $a \notin D_{\varepsilon}$, then

$$
n_{2 p-1}(r, a)-n_{2 p-1}(r, 0)<\varepsilon 2^{2 p-1}
$$

and

$$
n_{2 p}(r, a)-n_{2 p}(r, \infty)<\varepsilon 2^{2 p}
$$

for every $r>0$. Let $p>p_{\varepsilon}$ and $r_{2 p} \leq r<r_{2 p+1}=3 r_{2 p}$. Then we have

$$
n(r, 0)=\sum_{k=1}^{2 p-2} 2^{k}+n_{2 p-1}(r, 0)+n_{2 p+1}(r, 0)+2^{2 p}
$$

and for $w \notin D_{\varepsilon}$ 


$$
n(r, w)=\sum_{k=1}^{2 p-2} 2^{k}+n_{2 p-1}(r, w)+n_{2 p+1}(r, w)+n_{2 p}(r, w) .
$$

Now it follows from (4) and (5) that $n(r, w) \leq n(r, 0)-2^{2 p}+n_{2 p}(r, \infty)+$ $4 \varepsilon 2^{2 p}$ and we see from $(3)$ that $n(r, w) \leq n(r, 0)(1-1 / 80+4 \varepsilon)$ for $w \notin D_{\varepsilon}$. If $r_{2 p-1} \leq r<r_{2 p}$, we see in the same manner as above that $n(r, w) \leq n(r, \infty)(79 / 80+4 \varepsilon)$ for $w \notin D_{\varepsilon}$. Therefore we have $A(r) \leq$ $n(r)\left(79 / 80+4 \varepsilon+30 \varepsilon^{2}\right)$ for all large values of $r$ and we get $\lim \inf _{r \rightarrow \infty} n(r) / A(r) \geq 80 / 79$. Theorem 1 is proved.

6. Proof of Theorem 2. Let $q_{1}, q_{2}, \ldots$ be the sequence of all rational numbers on the segment $[0,2 \pi]$. We denote by $\varepsilon_{n}(z)$ a function satisfying the condition $\left|\varepsilon_{n}(z)\right|<1 / n$. We choose a sequence $t_{n}$ of positive integers such that $\lim _{n \rightarrow \infty} t_{n+1} / t_{n}=\infty$ and set

$$
f(z)=z \prod_{n=1}^{\infty} f_{n}(z)
$$

where

$$
f_{n}(z)=\prod_{k=1}^{t_{n}} \frac{1-z / r_{n, k}}{1-z /\left(r_{n, k}+\delta_{n}\right)} .
$$

Here $r_{n, k}=r_{n} \exp \left\{i k /\left(n^{2} t_{n}\right)\right\}, k=1, \ldots, t_{n},\left|\delta_{n}\right|=1$ and $\arg \delta_{n}=$ $\arg A_{n}-q_{n}$ where

$$
A_{n}=\prod_{p=1}^{n-1} \prod_{k=1}^{t_{p}}\left(1+\delta_{p} / r_{p, k}\right) .
$$

We assume that $r_{n} \rightarrow \infty$ as $n \rightarrow \infty$ so rapidly that

$$
f(z)=\left(1+\varepsilon_{n}(z)\right) r_{n} A_{n}\left(r_{n, k}-z\right) / \delta_{n}
$$

in every $C_{n, k}:\left|z-r_{n, k}\right|<r_{n}^{-1 / 2}, n \geq 2$, that there exists a finite limit $\lim _{n \rightarrow \infty} A_{n}=A \neq 0$, and that $f(z) \rightarrow \infty$ as $z \rightarrow \infty$ outside the union of the $\operatorname{discs} C_{n, k}$.

Let $w \neq \infty$. It follows from Rouché's theorem that if $n$ is sufficiently large, $f$ takes the value $w$ exactly once in every $C_{n, k}$. We choose an increasing sequence $n_{s}$ such that $\lim _{s \rightarrow \infty} \arg \delta_{n_{s}}=\pi$. For large values of $s$ we have

$$
n\left(r_{n_{s}}-1 / 2, w, f\right)=1+\sum_{k=1}^{n_{s}-1} t_{k}<2 t_{n_{s}-1}
$$

and $n\left(r_{n_{s}}-1 / 2, \infty, f\right)>t_{n_{s}}$. This implies that

$$
\lim \sup _{r \rightarrow \infty} n(r, \infty) / n(r, w)=\infty .
$$


Similarly, choosing the sequence $n_{s}$ such that $\lim _{s \rightarrow \infty} \arg \delta_{n_{s}}=0$, we see that $\lim \sup _{r \rightarrow \infty} n(r, w) / n(r, \infty)=\infty$.

Let $a$ and $b$ be finite, $a \neq b$, and $|a| \geq|b|$. For large values of $n$ we choose $a_{n, k}, b_{n, k} \in C_{n, k}$ such that $f\left(a_{n, k}\right)=a$ and $f\left(b_{n, k}\right)=b$. It follows from (6) that

$$
r_{n, k}-w_{n, k}=\delta_{n} w /\left(\left(1+\varepsilon_{n}\left(w_{n, k}\right)\right) A_{n} r_{n}\right)
$$

for $w=a, b$. We denote

$$
d_{n}=\frac{|a|-|a-b|^{2} /(32|a|)}{\left|A_{n}\right| r_{n}} .
$$

We choose an increasing sequence $n_{s}$ such that $\lim _{s \rightarrow \infty} q_{n_{s}}=\arg a$. Then $\lim _{s \rightarrow \infty} \arg \left(r_{n_{s}, k}-a_{n_{s}, k}\right)=0$ and we see from (7) that $\left|a_{n_{s}, k}\right|<r_{n_{s}}-d_{n_{s}}$ $<\left|b_{n_{s}, k}\right|$ for all large values of $s$. This implies that

$$
\lim \sup _{r \rightarrow \infty} n(r, a) / n(r, b)=\infty .
$$

If the sequence $n_{s}$ is chosen such that $\lim _{s \rightarrow \infty} q_{n_{s}}=\pi+\arg \alpha$, then we have $\left|b_{n_{s}, k}\right|<r_{n_{s}}+d_{n_{s}}<\left|a_{n_{s}, k}\right|$ for all large values of $s$. Therefore $\lim \sup _{r \rightarrow \infty} n(r, b) / n(r, a)=\infty$. This completes the proof of Theorem 2 .

7. Proof of Theorem 3. Let $q_{n}$ and $\varepsilon_{n}(z)$ be as in the proof of Theorem 2. We choose a sequence $t_{n}$ of positive integers such that $\lim _{n \rightarrow \infty} t_{n} / t_{n-1}^{4}=\infty$. For each $n$ we choose $\delta_{n}, 1<\delta_{n}<1+1 / n$, such that the polynomial

$$
g(z)=\prod_{k=1}^{t_{n}^{2}}\left(1-z / b_{k}\right)
$$

where $b_{k}=\exp \left\{2 \pi i k / t_{n}^{2}\right\}, k=1, \ldots, t_{n}$, and $b_{k}=\delta_{n} \exp \left\{2 \pi i k / t_{n}^{2}\right\}$ for $k=t_{n}+1, \ldots, t_{n}^{2}$, satisfies the condition $g(z)=\left(1+\varepsilon_{t_{n}}(z)\right)\left(1-z^{t_{n}^{2}}\right)$ in

$$
\left\{z: \pi / t_{n}^{2} \leq \arg z \leq 2\left(t_{n}+1 / 2\right) \pi / t_{n}^{2}, \quad 1 / 2 \leq|z| \leq 2\right\} .
$$

We set

$$
f(z)=\prod_{n=1}^{\infty} f_{n}(z) \text { and } f_{n}(z)=\prod_{k=1}^{t_{n}^{2}}\left(1-z / r_{n, k}\right)
$$

where $\quad r_{n, k}=r_{n} \exp \left\{2\left(p_{n}+k\right) \pi i / t_{n}^{2}\right\}, \quad k=1, \ldots, t_{n}, \quad$ and $r_{n, k}=$ $r_{n} \delta_{n} \exp \left\{2\left(p_{n}+k\right) \pi i / t_{n}^{2}\right\}$ for $k=t_{n}+1, \ldots, t_{n}^{2}$. We denote

$$
A_{n}=\prod_{s=1}^{n-1} \prod_{k=1}^{t_{s}^{2}}\left(-r_{s, k}\right)^{-1} \text { and } s_{n}=\sum_{s=1}^{n-1} t_{s}^{2} \text {. }
$$

Here $p_{n}$ is the smallest positive integer such that $\arg A_{n} z^{\varsigma_{n}}=q_{n}$ for some 
$z$ satisfying the condition $2 \pi p_{n} / t_{n}^{2} \leq \arg z<2 \pi\left(p_{n}+1\right) / t_{n}^{2}$. We assume that $r_{n} \rightarrow \infty$ as $n \rightarrow \infty$ so rapidly that $f(z) \rightarrow \infty$ as $z \rightarrow \infty$ outside the union of the dises $C_{n, k}:\left|z-r_{n, k}\right|<1 / r_{n}$, and for every $n \geq 2$, $r_{n}\left|A_{n}\right|>1$ and $r_{n}\left(\delta_{n}-1\right)>1$. Furthermore, we may assume that

$$
f(z) / f_{n}(z)=\left(1+\varepsilon_{4 n}(z)\right) A_{n} z^{s_{n}}
$$

in $r_{n}|2<| z \mid<2 r_{n}$ and

$$
\begin{aligned}
f_{n}(z) & =\left(1+\varepsilon_{t_{n}}(z)\right)\left(1-\left(z / r_{n}\right)^{t_{n}^{2}}\right) \\
& =t_{n}^{2}\left(1+\varepsilon_{4 n}(z)\right)\left(r_{n, k}-z\right) / r_{n, k}
\end{aligned}
$$

in $C_{n, k}, \quad 1 \leq k \leq t_{n}, n \geq 2$.

Let $a \neq \infty$. If $n$ is sufficiently large, say $n \geq n_{0}$, there exists exactly one point $a_{n, k} \in C_{n, k}$ such that $f\left(a_{n, k}\right)=a$. It follows from (8) and (9) that

$$
r_{n, k}-a_{n, k}=a\left(1+\varepsilon_{n}\left(a_{n, k}\right)\right) /\left(\left|A_{n}\right| r_{n}^{s_{n}-1} t_{n}^{2} \exp \left\{i q_{n}\right\}\right)
$$

for $n>n_{0}, k=1, \ldots, t_{n}$.

Let $a$ and $b$ be finite, $a \neq b$. As in the proof of Theorem 2, we see from (10) that there exist arbitrarily large values of $n$ such that for some $\varrho_{n}, r_{n}-1 / r_{n}<\varrho_{n}<r_{n}+1 / r_{n}$, we have $n\left(\varrho_{n}, a, f\right)>t_{n}$ and $n\left(\varrho_{n}, b, f\right)<2 t_{n-1}^{2}$. Therefore $\lim \sup _{r \rightarrow \infty} n(r, a) / n(r, b)=\infty$, and Theorem 3 is proved.

8. Proof of Theorem 4. We denote $r_{n}=\exp \left\{(2 s)^{n}\right\}$ and $\varrho_{n}=r_{n}^{1+1 /(2 s)}$. Let $n \geq 8$ be even. Let $|z|=\varrho_{n}$. We have $\log |1-z| r_{n-1} \mid \geq \log r_{n}-$ $2 / r_{n}$ and $\log |1-z| r_{n-2} \mid \leq(1+1 /(2 s)) \log r_{n}$. Therefore we get

$$
\log \left|\prod_{m=1}^{n-1}\left(1-z / r_{m}\right)^{(-s)^{m}}\right| \leq-s^{n-1}(1-2 / s) \log r_{n} .
$$

Furthermore, we have $\log \left|\left(1-z / r_{n}\right)^{s^{n}}\right| \leq\left(3 s^{n-1} / 5\right) \log r_{n}$ and

$$
\log \left|\prod_{m=n+1}^{\infty}\left(1-z / r_{m}\right)^{(-s)^{m}}\right| \leq 1 .
$$

Combining these estimates we see that $|f(z)|<1 / r_{n}$ on the circle $|z|=\varrho_{n}$, if $n$ is even, $n \geq 8$. Similarly, if $n \geq 9$ is odd then $|f(z)|>r_{n}$ on $|z|=\varrho_{n}$.

Let $p \geq 5$. It follows from Rouché's theorem that $n\left(\varrho_{2 p}, a\right)=$ $n\left(\varrho_{2 p}, \infty\right)$ for $|a| \geq 1 / r_{2 p}$, and if $|a|<1 / r_{2 p}$ then $n\left(\varrho_{2 p}, a\right) \leq$ $n\left(\varrho_{2 p+1}, a\right)=n\left(\varrho_{2 p+1}, 0\right)$. Therefore

$$
A\left(\varrho_{2 p}\right) \leq n\left(\varrho_{2 p}, \infty\right)+n\left(\varrho_{2 p}, 0\right) / r_{2 p}<2 s^{2 p-1}
$$

and we see that $n\left(r_{2 p}, 0\right)>(s / 2) A\left(\varrho_{2 p}\right)$. This implies that for all 


$$
r \in B=\bigcup_{p=5}^{\infty}\left[r_{2 p}, r_{2 p}^{1+1 /(5 s)}\right]
$$

we have $n(r, 0) / A\left(r^{1+1 /(5 s)}\right)>s / 2$. Clearly $B$ has lower logarithmic density at least $(2 s)^{-4}$. Theorem 4 is proved.

9. Proof of Theorem 5. Let $n \geq 4$. We have $|f(z)|<1 / r_{n}$ on $\left|z-r_{n}\right|=3 r_{n} / 4$ and $|f(z)|>r_{n}$ on the circles $\left|z-r_{n}\right|=11 r_{n} / 12$ and $|z|=r_{n} / 12$. Then it follows from Rouché's theorem that $n\left(r_{n} / 12, a\right)=$ $n\left(r_{n} / 12,0\right)$ for $|a|<r_{n}$. The function $\log f(z), \arg f\left(11 r_{n} / 6\right)=0$, is analytic in

$$
D_{n}=\left\{z: 3 r_{n} / 4 \leq\left|z-r_{n}\right| \leq 11 r_{n} / 12, \operatorname{Re} z \geq r_{n}\right\}
$$

and if $3 r_{n} / 4 \leq y \leq 11 r_{n} / 12$, then $\arg f\left(r_{n}+i y\right)>\pi t_{n} / 2$ and $\arg f\left(r_{n}-i y\right)<-\pi t_{n} / 2$. Therefore $f$ takes every value $a$ satisfying $1 / r_{n}<|a|<r_{n}$ at least $t_{n} / 2$ times in $D_{n}$. The disc $|z| \leq 7 r_{n} / 6$ does not contain any point of $D_{n}$, and we see that

$$
n\left(7 r_{n} / 6, a\right)<t_{n} / 2+\sum_{k=1}^{n-1} t_{k}
$$

for $1 / r_{n}<|a|<r_{n}$. We have $M\left(2 r_{n}, f\right)<\min _{|z|=r_{n}{ }^{2}}|f(z)|$ and therefore $n\left(2 r_{n}, a\right)<2 t_{n}$ for any $a \in \Sigma$. Combining these estimates we get

$$
A\left(7 r_{n} / 6\right)<t_{n} / 2+\sum_{k=1}^{n-1} t_{k}+4 t_{n} / r_{n} .
$$

Because $n\left(r_{n}, 0\right)>t_{n}$, we see now that $n\left(r_{n}, 0\right)>(9 / 5) A\left(7 r_{n} / 6\right)$ for $n \geq 4$. Theorem 5 is proved.

10. Proof of Theorem 6 . Let $K \geq 1$. Let $n \geq 4$ be so large that $s_{n}=r_{n} /(8 K)>9 r_{n-1}$. We denote by $D$ the bounded domain bounded by the lines $L_{1}: \operatorname{Re} z=s_{n}, L_{2}: \operatorname{Re} z=3 s_{n}, L_{3}: \operatorname{Im} z=s_{n}$, and $L_{4}: \operatorname{Im} z=-s_{n}$. The boundary of $D$ is denoted by $T$. The function $\log f(z), \arg f(1)=0$, is regular in $\operatorname{Re} z>0$, and we see that $|\arg f(z)|>$ $s_{n} t_{n} /\left(2 r_{n}\right)$ on $\left(L_{3} \cup L_{4}\right) \cap \Gamma,|f(z)|<f\left(2 s_{n}\right)$ on $L_{1} \cap \Gamma$, and $|f(z)|>$ $f\left(2 s_{n}\right)$ on $L_{2} \cap T$. Therefore $\log f(z)$ takes in $D$ all values $\log f\left(2 s_{n}\right)+$ $i y,|y| \leq s_{n} t_{n} /\left(2 r_{n}\right)$. This implies that

$$
n\left(4 s_{n}\right) \geq t_{n} /(32 \pi K) \text {. }
$$

We see easily that $f(z) / z \rightarrow \infty$ as $z \rightarrow \infty$ outside the union of the discs $C_{n}:\left|z+r_{n}\right|<r_{n} / 12$. If $n$ is large then $n\left(r_{n} / 2, a\right)=$ $=n\left(r_{n} / 2,0\right)<2 t_{n-1}$ for $|a|<r_{n}$. Because $M\left(r_{n}, f\right)<\min _{|z|=r_{n}^{2}}|f(z)|$, 
we have $n\left(r_{n}, a\right) \leq n\left(r_{n}^{2}, 0\right)<2 t_{n}$ for every $a \in \Sigma$. Therefore

$$
A\left(r_{n} / 2\right)<2 t_{n-1}+8 t_{n} / r_{n}<3 t_{n-1}
$$

for all large values of $n$. Combining (11) and (12), we see that $\lim \sup _{r \rightarrow \infty} n(r) / A(K r)=\infty$. Theorem 6 is proved.

\section{References}

[1] Hayman, W. K.: New problems. - Symposium on Complex Analysis, Canterbury, 1973, Clunie, J. and W. K. Hayman eds., London Math. Soc. Lecture Note Series 12, Cambridge University Press, Cambridge, 1974, 155- 180.

[2] -"- Meromorphic functions. - Clarendon Press, Oxford, 1964.

[3] - - - Research problems in function theory. - Athlone Press, London, 1967.

[4] Hayman, W. K., and F. M. STEWART: Real inequalities with applications to function theory. - Proc. Cambridge Philos. Soc. 50, 1954, 250-260.

[5] MrLES, J.: Bounds on the ratio $n(r, a) / S(r)$ for meromorphic functions. Trans. Amer. Math. Soc. 162, 1971, 383-393.

[6] Rickman, S.: On the value distribution of quasimeromorphic maps. - Ann. Acad. Sci. Fenn. Ser. A I 2, 1976, 447-466.

University of Helsinki

Department of Mathematics

SF-00100 Helsinki 10

Finland.

Received 29 August 1975 